\title{
On the Correlation Function of an Arbitrary Distributed Continuous Markov Process
}

\author{
V. LYANDRES \\ School of Electrical and Computer Engineering, \\ Ben-Gurion University of the Negev, \\ P.O. Box 653, Beer-Sheva, 84105 \\ ISRAEL
}

\begin{abstract}
Continuous Markov processes widely used as a tool for modeling random phenomena in numerous applications, can be defined as solutions of generally nonlinear stochastic differential equations (SDEs) with certain drift and diffusion coefficients which together governs the process' probability density and correlation functions. Usually it is assumed that the diffusion coefficient does not depend on the process' current value. Sometimes, in particular for presentation of nonGaussian real processes this assumption becomes undesirable, leads generally to complexity of the correlation function estimation. We consider its analysis for the process with arbitrary pair of the drift and diffusion coefficients providing the given stationary probability distribution of the considered process.
\end{abstract}

Keywords: Continuous Markov process; Stochastic differential equation; Fokker-Planck equation; Galerkin method

Received: April 28, 2019. Revised: April 21, 2020. Accepted: April 30, 2020. Published: May 8, 2020

\section{Introduction}

There is a wide range of phenomena in economics (stock prices) [1, 2], biology (population, epidemics) [3, 4], радио physics [5] that can be modeled as diffusion (continuous-time) Markov processes, which in turn, are represented by solutions of certain first-order stochastic differential equations (SDEs) characterized by the drift $K_{1}(x)$ and diffusion $K_{2}(x)$ coefficients. For some of them, those coefficients depend on their current values, and for such diffusion Markov process their generating SDE is written as [6]

$$
\dot{x}=K_{1}(x)+\sqrt{K_{2}(x)} \xi(t)
$$

where $\xi(t)$ is White Gaussian Noise (WGN) with correlation function

$$
<\xi(t) \xi(t+\tau)>=\delta(\tau) .
$$

Our purpose is to find an approximate method of calculation the correlation function for the continuous Markov process with the given stationary probability density and arbitrary diffusion coefficient. The paper is organized as follows: some preliminary results are presented in Section 2. Exact solution, i.e. spectrum of eigenvalues and expression for eigenfunctions of the FokkerPlanck equation (FKE) corresponding to SDE (1), known only for some particular pairs of functions $K_{1}(x)$ and $K_{2}(x)$, is considered in Section 3. Section 4 is devoted to $\mathrm{n}$ application of the Galerkin method for analysis of the correlation properties of the process with arbitrary pair of aforementioned functions.

\section{Preliminary results}

Using the Stratonovich definition of stochastic integral [6] we can express the drift coefficient $K_{1}(x)$ as a function of the process $x(t)$ stationary density $f_{s t}(x)$ and $K_{2}(x)$

$$
K_{1}(x)=f_{s t}(x)-\frac{1}{4} K_{2}(x)
$$

and the density $f_{s t}(x)$ depends on $K_{1}(x)$ and $K_{2}(x)$ as

$$
f_{s t}(x)=\frac{C}{\sqrt{K_{2}(x)}} \exp \left[2 \int_{-\infty}^{x} \frac{K_{1}(z)}{K_{2}(z)} d z\right] .
$$

For a given $f_{s t}(x)$, solving (4) with respect to $K_{1}(x)$ we obtain 


$$
K_{1}(x)=\frac{1}{2}\left\{\begin{array}{l}
K_{2}(x) \frac{d}{d x} \ln f_{s t}(x)+ \\
\sqrt{K_{2}(x)} \frac{d}{d x}\left[\sqrt{K_{2}(x)}\right]
\end{array}\right\} .
$$

According to (4) the prescribed stationary density $f_{s t}(x)$ can be obtained for SDE with any diffusion coefficient.

To find the correlation function of a stationary continuous Markov process the parabolic type Fokker-Planck equation (FKE) for the probability density $f[x, t]$ of the process has to be solved. One of the methods of the FKE solution $[4,6]$ is based on the presentation of this density as

$$
f[x, t]=Y(x) T(t) .
$$

Substituting (6) in the FKE [6]

$$
\begin{aligned}
& \frac{\partial}{\partial t} f(x, t)=-\frac{\partial}{\partial x}\left[K_{1}(x) f(x, t)\right]+ \\
& \frac{1}{2} \frac{\partial^{2}}{\partial x^{2}}\left[K_{2}(x) f(x, t)=0\right.
\end{aligned}
$$

we obtain the following transformation

$\frac{1}{T(t)} \frac{\partial}{\partial t} T(t)=$

$$
\frac{-\frac{\partial}{\partial x}\left[K_{1}(x) Y(x)\right]+\frac{1}{2} \frac{\partial^{2}}{\partial x^{2}}\left[K_{2}(x) Y(x)\right]}{Y(x)}=0
$$

which, in turn, is equivalent to the system of two ordinary differential equations

$$
\begin{aligned}
& \frac{1}{T(t)} \frac{d}{d t} T(t)=-\lambda, \quad \lambda \geq 0, \\
& \frac{1}{2} \frac{d^{2}}{d x^{2}}\left[K_{2}(x) Y(x)\right]- \\
& \frac{d}{d x}\left[K_{1}(x) Y(x)\right]+\lambda Y(x)=0,
\end{aligned}
$$

and the solution of the FKE may be written as

$$
f(x, t)=\sum_{n=0}^{\infty} Y_{n}\left(x_{n}, \lambda_{n}\right) \exp \left(-\lambda_{n} t\right) .
$$

Here $\lambda_{n}$ and $Y_{n}(x)$ are real eigenvalues and eigenfunctions of (10) corresponding to the boundary conditions

$$
Y_{n}(0)=Y_{n}(\infty)=0
$$

or

$$
Y_{n}(-\infty)=Y_{n}(\infty)=0,
$$

depending on the process range.
The eigenvalue $\lambda_{0}=0$ is related to the stationary solution of the FKE $f_{s t}(x)$. If the initial value of the process is fixed, i.e.

$$
p(x, t=0)=\delta\left(x-x_{0}\right),
$$

the transitional density of the process is written in the following form [5]

$$
\begin{aligned}
& \pi\left(x, t / x_{0}, t_{0}\right)= \\
& \sum_{n=0}^{\infty} \frac{Y_{n}(x) Y_{n}\left(x_{0}\right)}{f_{s t}(x)} \exp \left[-\lambda_{n}\left(t-t_{0}\right)\right]
\end{aligned}
$$

while the correlation function is expressed as

$$
B(\tau)=\sum_{n=0}^{\infty} h_{n}^{2} \exp \left[-\lambda_{n}|\tau|\right],
$$

where

$$
h_{n}=\int_{-\infty}^{\infty} x Y_{n}(x) f_{s t}(x)
$$

\section{Correlation function of the continuous Markov process with particular pair of the drift and diffusion coefficients}

As is clear from (16) the correlation function of the diffusion Markov process is monotonically decreasing but its convergence to an approximately exponential function depends on the set of functions $\left\{Y_{n}(x)\right\}$ and on the spectrum of eigenvalues $\left\{\lambda_{n}\right\}$ of the equation (10) considering (5) can be rewritten as

$$
\begin{gathered}
\frac{d^{2} Y_{n}(x)}{d x^{2}}+\frac{d \ln f_{s t}(x)}{d x} \frac{d Y_{n}(x)}{d x}+ \\
{\left[\begin{array}{l}
\frac{2 \lambda_{n}}{K_{2}(x)}-\frac{d \ln K_{2}(x)}{d x} \frac{d \ln f_{s t}(x)}{d x}- \\
\frac{d^{2} \ln f_{s t}(x)}{d x}
\end{array}\right] Y_{n}(x)=0}
\end{gathered}
$$

The closed form solution of (18) may be obtained only for special pair of $K_{2}(x)$ and $f_{s t}(x)$. In the case of the process with constant diffusion it is available for the Gaussian or the Nakagami-m density [7] (generalizing Rayleigh, Weibull, Maxwell, the single-sided normal and the multidimensional vector modulus distributions) 


$$
f_{s t}(x)=\left\{\begin{array}{c}
0, \quad-\infty<x<0 \\
\frac{2 m^{m}}{\Gamma(m)} \frac{x^{2 m-1}}{\sigma^{2 m}} \exp \left(-\frac{m x^{2}}{\sigma^{2}}\right), \quad x \geq 0, m \geq 0.5
\end{array}\right.
$$

For the Gaussian process generated by SDE with linear drift and constant diffusion all eigenvalues except the first are equal to zero and the correlation function is exactly exponential [5]. For densities of the group (19), the solution of (18) may be found in [7]

$$
\begin{aligned}
& Y_{n}(x)=2 \sqrt{\frac{n !}{\Gamma(m+n) \Gamma(m)}} \frac{x^{2 m-1}}{\sigma^{2 m}} \\
& \exp \left(-\frac{m x^{2}}{\sigma^{2}}\right) L_{n}^{(m-1)}\left(\frac{m}{\sigma^{2}}\right) \\
& \lambda_{n}=\frac{2 N_{0} m}{\sigma^{2}} n, \quad n=0,1,2, \ldots,
\end{aligned}
$$

where $L_{n}^{(m-1)}(z)$ is the generalized Laguerre polynomial [9]. The correlation function of such processes is represented by the row [8]

$$
\begin{aligned}
& B(\tau)=\frac{\sigma^{2} \Gamma^{2}(m+0.5)}{4 m \pi \Gamma(m)} \\
& \sum_{n=1}^{\infty} \frac{\Gamma^{2}(n-0.5)}{\Gamma(m+n) n !} \exp \left(-\frac{2 N_{0} m}{\sigma^{2}} n|\tau|\right)
\end{aligned} .
$$

In the case of Gamma distribution [10] generalizing Pearson, Erlang, Laplace, exponential power distributions

$$
\begin{aligned}
& f_{s t}(x)= \\
& \left\{\begin{array}{c}
0, \quad-\infty<x<0 \\
\frac{x^{\alpha}}{\beta^{\alpha+1}} \frac{x^{2 m-1}}{\Gamma(\alpha+1)} \exp \left(-\frac{x}{\beta}\right), x \geq 0, \alpha>-1, \beta>0
\end{array}\right.
\end{aligned}
$$

and constant diffusion coefficient, eigenfunctions of equation (18) cannot be expressed with the help of known functions, but this can be done if

$$
K_{2}(x)=N_{0} x .
$$

In this case its eigenfunctions are

$$
\begin{aligned}
& Y_{n}(x)=\sqrt{\frac{n !}{\Gamma(\alpha+1) \Gamma(\alpha+1+n)}} \\
& x^{\alpha} \exp \left(-\frac{x}{\beta}\right) L_{n}^{(\alpha)}\left(\frac{x}{\beta}\right)
\end{aligned}
$$

and the only nonzero eigenvalue is

$$
\begin{gathered}
\lambda_{1}=\frac{N_{0}}{2 \beta} \\
h_{1}=-\beta \sqrt{\Gamma(\alpha+1)},
\end{gathered}
$$

while the correlation function of the Gamma distributed Markov process with linear diffusion coefficient is

$$
B(\tau)=\beta^{2}(\alpha+1) \exp \left(-\frac{N_{0}}{2 \beta}|\tau|\right),
$$

which is identical to that of the Gaussian Markov process [6].

\section{Correlation function of the continuous Markov process with arbitrary pair of the drift and diffusion coefficients}

In [8] it was shown that for a process with constant diffusion and symmetrical density close to Gaussian the correlation function may be approximately presented by an exponential form

$$
B(\tau) \approx M_{2 x} \exp \left[-\left(a_{1}+a_{3} b\right) \tau \mid\right],
$$

where

$$
\begin{gathered}
a_{1}=\left[f_{s t}(x)+\frac{1}{2} \sqrt{K_{2}(x)}\right]_{x=0}, \\
a_{3}=\frac{1}{2}\left[\frac{d^{2}}{d x^{2}} f_{s t}(x)+\frac{1}{2} \frac{d^{2}}{d x^{2}} \sqrt{K_{2}(x)}\right]_{x=0}, \\
b=\frac{M_{4 x}}{M_{2 x}} .
\end{gathered}
$$

To find correlation function of the process generated by SDE (1) with arbitrary pair of drift and diffusion we consider here the Galerkin method [11] for the solution of equation (18). The important feature of this method is that it provides uniform convergence to the exact solution that is being looked in the form

$$
Y(x) \approx Y^{(p)}=\sum_{k=1}^{p} a_{n} \varphi_{k}(x),
$$

where $\left\{a_{k}, \quad k=1,2, . ., p\right\}$

are undetermined coefficients and $\left\{\varphi_{k}(x)\right\}$ is the complete system of chosen beforehand mutually independent functions satisfying the boundary conditions (12) or (13). In the first case, these functions may be polynomials

$$
L_{p}(x)=\sum_{k=1}^{p-1} a_{k}\left(\frac{x}{x+1}\right)^{k},
$$

and for the case of the boundary conditions (13) polynomials 


$$
L_{p}(x)=\sum_{k=1}^{p-1} a_{n}\left(\frac{x^{2}}{x^{2}+1}\right) .
$$

Multiplying $\varphi_{k}(x)$ in (34) by $\frac{1}{x+1}$ and $\varphi_{k}(x)$ in (35) by $\frac{1}{x^{2}+1}$, instead of (34) we obtain

$$
Y^{(p)}(x)=\sum_{k=1}^{p} a_{k} \frac{x^{k-1}}{(x+1)^{k}},
$$

and instead of (35) we obtain

$$
Y^{(p)}(x)=\sum_{k=1}^{p} a_{n} \frac{x^{2(k-1)}}{\left(x^{2}+1\right)^{k}} .
$$

The systems of functions

$$
\varphi_{k}(x)=\frac{x^{k-1}}{(x+1)^{k}}
$$

and

$$
\varphi_{k}(x)=\frac{x^{2(k-1)}}{\left(x^{2}+1\right)^{k}}
$$

are complete, i.e. for any function $Y_{n}^{(p)}(x)$ continuous with its derivative and satisfying (12) or (13) the following linear form exists

$$
\sum_{k=1}^{p} a_{k} \varphi_{k}(x)
$$

that

$$
\left|Y^{(p)}(x)-\sum_{k=1}^{p} a_{k} \varphi_{k}(x)\right|<\varepsilon,
$$

and

$$
\left|\left[Y^{(p)}(x)\right]^{\prime}-\sum_{k=1}^{p} a_{k} \varphi_{k}^{\prime}(x)\right|<\varepsilon,
$$

where $\varepsilon$ is an arbitrary small positive number.

Denoting

$$
z=\frac{x}{x+1}
$$

or

$$
z=\frac{x^{2}}{x^{2}+1},
$$

we obtain the system complete at the interval $[0,1]$ and common for both cases of bounded conditions

$$
\varphi_{k}(z)=(1-z) z^{k-1} .
$$

Let us write, conforming to [9], the system of linear equations for coefficients $a_{k}$

$$
\sum_{k=1}^{p}\left(\alpha_{k l}+\lambda \gamma_{k l}\right) a_{k}=0, l=1,2, \ldots, p,
$$

where the term in brackets are

$$
\alpha_{k l}=\int_{-\infty}^{\infty}\left\{\begin{array}{c}
\frac{d^{2} \varphi(x)}{d x^{2}}+\left[\frac{d}{d x} \ln \frac{K_{2}(x)}{f_{s t}(x)}\right] \frac{d \varphi_{k}(x)}{d x}- \\
{\left[\frac{d}{d x} \ln K_{2}(x) \frac{d f_{s t}(x)}{d x}\right]-} \\
{\left[\begin{array}{l}
\frac{d}{d x} \ln K_{2}(x) \frac{d f_{s t}(x)}{d x}+ \\
\frac{d^{2}}{d x^{2}} \ln f_{s t}(x) \varphi_{k}(x)
\end{array}\right] \varphi_{k}(x)} \\
\varphi_{l}(x) d x
\end{array}\right\}
$$

and

$$
\gamma_{k l}=2 \int_{-\infty}^{\infty} \frac{\varphi_{k}(x) \varphi_{l}(x)}{K_{2}(x)} d x .
$$

Substituting in (47) and (48) expressions for $\varphi_{k}(x)$ and its first and second derivatives,

$$
\frac{d \varphi_{k}(x)}{d x}=\frac{x^{k-2}(k-x-1)}{(x+1)^{k+1}}
$$

and

$$
\frac{d^{2} \varphi_{k}(x)}{d x^{2}}=\frac{x^{k-3}\left(-4 k x+2 x^{2}+4 x+k^{2}-3 k\right)}{(x+1)^{k+2}}
$$

or

$$
\frac{d \varphi_{k}(x)}{d x}=\frac{2 x^{2 k-3}\left(k-x^{2}-1\right)}{\left(x^{2}+1\right)^{k+1}}
$$

and

$$
\begin{aligned}
& \frac{d^{2} \varphi_{k}(x)}{d x^{2}}= \\
& \frac{\left.2 x^{2 k-4}\left(2 k^{2}-7 k x^{2}-5 k\right)+3 x^{4}+6 x^{2}+3\right)}{\left(x^{2}+1\right)^{k+2}}
\end{aligned}
$$

we obtain for the boundary conditions (12) the following expressions for the coefficients $\alpha_{k l}$ and $\gamma_{k l}$ 


$$
\begin{aligned}
& \alpha_{k l}= \\
& \int_{-\infty}^{\infty}\left\{\begin{array}{c}
\frac{x^{k-3}\left(-4 k x+2 x^{2}+4 x+k^{2}-3 k\right)+2}{(x+1)^{k+2}} \\
+\left[\frac{d}{d x} \ln \frac{K_{2}(x)}{f(x)}\right] \frac{x^{k-2}(k-x-1)}{(x+1)^{k+1}} \\
-\left[\frac{d}{d x} \ln K_{2}(x) \frac{d}{d x} \ln f(x)+\frac{d^{2}}{d x^{2}} \ln f(x)\right] \frac{x^{k-1}}{(x+1)^{k}}
\end{array}\right\}
\end{aligned}
$$

and

$$
\gamma_{k l}=2 \int_{0}^{\infty} \frac{x^{k+l-2}}{K_{2}(x)(x+1)^{k+l}} d x,
$$

For the bounded conditions (13) we have

$$
\alpha_{k l}=\int_{-\infty}^{\infty}\left\{\begin{array}{c}
\frac{2 x^{2 k-4}\left(2 k^{2}-7 k x^{2}-5 k+3 x^{4}+6 x^{2}+3\right)}{\left(x^{2}+1\right)^{k+2}} \\
\left.+\left[\frac{d}{d x} \ln \frac{K_{2}(x)}{f(x)}\right] \frac{2 x^{2 k-3}\left(k-x^{2}-1\right)}{\left(x^{2}+1\right)^{k+1}} \ln K_{2}(x) \frac{d}{d x} \ln f(x)+\frac{d^{2}}{d x^{2}} \ln f(x)\right] \frac{2 x^{2 k-1}}{\left(x^{2}+1\right)^{k}}
\end{array}\right\}
$$

and

$$
\gamma_{k l}=2 \int_{0}^{\infty} \frac{x^{2(k+l-2)}}{K_{2}(x)\left(x^{2}+1\right)^{k+l}} d x .
$$

For a given diffusion $K_{2}(x)$ and stationary density $f_{s t}(x)$ we can write the determinant of the $p-$ th order

$$
\operatorname{Det}\left\|\alpha_{k l}+\gamma_{k l}\right\|, \quad(k, l \leq p), \quad k, l \leq p .(57)
$$

If it equals zero we obtain the equation of the $p$-th order regarding $\lambda$ with $p$ positive roots $\lambda_{1}^{[p]}, \lambda_{2}^{[p]}, \ldots, \lambda_{i}^{[p]}, \ldots, \lambda_{p}^{[p]}$ representing approximate values of eigenvalues, and the approximation of eigenfunctions may be written as

$$
Y_{n}^{[p]}(x) \approx \sum_{k=1}^{p} C_{n}^{[p]} a_{k}^{(n)} \varphi_{k}(x),
$$

where constant values $\left\{C_{n}^{[p]}\right\}$ can be found with the help of normalization condition

$$
\int_{-\infty}^{\infty} \frac{\left[C_{i}^{[p]} Y_{i}^{[p]}\right]^{p}}{f_{1}(x)} d x=1
$$

Calculating for any $i \leq p$

$$
h_{i}^{[p]}=\int_{-\infty}^{\infty} C_{i}^{[p]} Y_{i}^{[p]}(x) x d x
$$

and substituting the resulting expression in (15), we obtain the approximate expression of the correlation function of the continuoustime Markov process with the given stationary density and diffusion coefficient

$$
B_{x}^{[p]}(\tau)=\sum_{i=1}^{p}\left(h_{i}^{(p)}\right)^{2} \exp \left(-\lambda_{i}^{(p)}|\tau|\right) .
$$

\section{Conclusions}

Continuous Markov process may be presented as a solution of a generally nonlinear stochastic differential equation (SDE). The process' properties, including stationary probability distribution and correlation function, depend on its drift and diffusion forming the operator of SDE. The required process' density may be obtained with the help of their different pairs, while the correlation function appears as an infinite sum of exponents with the decays to be found from the corresponding Fokker-Planck equation with certain boundary conditions. Its closed form solution may be obtained In this paper we have consider analysis of the correlation function of the continuous Markov process, which consists in trying to solve the corresponding Fokker-Planck equation at certain boundary conditions, while its closed form solution rarely occurs. We consider process with arbitrary pair of drift and diffusion assuring its required density, and apply the approximate Galerkin method which guaranties uniform convergence to the exact expression of correlation function

\section{References}

[1] W.J. Hull, Futures and other Derivatives, Pearson Education India, 2006. 
[2] E. Nicolato, E. Venardos, Option pricing in stochastic volatility models of the Ornstein-Uhlenbeck type, Mathematical Finance, 13 (4) (2003) 445-466.

[3] P. E. Smouse, S. Focardi, P. R. Moorcroft, J. G. Kie, J. D. Forester, J. M. Morales, Stochastic modelling of animal movement, Philosophical Transactions of the Royal Society B: Biological Sciences, 365 (1550) 2201-2211.

[4] A. T. Barucha-Reid, Elements of the Theory of Markov Processes and their Applications, McGraw Hills, 1960.

[5] V. Lyandres, Model of deep fading, Information and Control Systems, 1 (2018) 123-127.

[6] R. L. Stratonovich, Topics in the Theory of Random Noise, Vol. 1, Gordon and Breach, 1967.
[7] M. Nakagami, The m-distribution - A general formula of intensity of rapid fading, Methods in Radio Wave Propagation, Pergamon, 1960.

[8] S. Primak, V. Kontorovich, V. Lyandres, Stochastic Methods and their Applications to Communications. Stochastic Differential Equations Approach, Wiley, 2004.

[9] I. Gradshtein, I. Ryzhik, Tables of Integrals, Series, and Products, Academic Press, 2014.

[10] A. Zayezdny, D. Tabak, D. Wulich, Engineering Applications of Stochastic Processes: Theory, Problems and Solutions, Research Studies Press, Tauton, Somerset, 1989.

[11] L. V. Kantorovich, V. I. Krylov, Approximate Methods of Higher Analysis, InterScience, 1964 